\title{
Hybrid WDM-TDM PON Architectures and DWBA Algorithms
}

\author{
Luying Zhou, Xiaofei Cheng, Yong-Kee Yeo, Lek Heng Ngoh \\ Institute for Infocomm Research, A*STAR, Singapore 138632 \\ Email: \{lzhou, chengxf, ykyeo, lhn\}@i2r.a-star.edu.sg
}

\begin{abstract}
Passive optical networks (PONs) represent one of the most attractive access network solutions. TDM and WDM techniques are employed in the PON for higher resource efficiency and capacity, which results in TDM PON and WDM PON respectively. Hybrid WDM-TDM PON combines the advanced features of both TDM PON and WDM PON, and provides a more flexible and cost-effective access network solution. We review the current work in the hybrid WDM-TDM PON architectures, dynamic wavelength and bandwidth allocation (DWBA) algorithms and enabling technologies, and present the development and deployment trends from TDM PON to the hybrid WDM-TDM PON. We analyze the deployment problem incurred under physical constraints, and point out there is a need to develop DWBA algorithms with both upstream and downstream traffic scheduling taken into consideration.

Index Terms - TDM PON, WDM POM, Hybrid WDM-TDM PON, Architectures, DWBA Algorithm
\end{abstract}

\section{INTRODUCTION}

Passive Optical Networks (PONs) have been considered as a promising access network solution due to the high bandwidth provision, and the low operation and maintenance cost. There are various PONs specified by different standard organizations, i.e., APON (ITU-T G.983.1), BPON (ITU-T G.983.4), EPON (IEEE 802.3ah) and GPON (ITU-T G.984). Currently the widely deployed PONs are EPON (in Asia countries) and GPON (in Europe, North America). EPON - Ethernet PON, which transports Ethernet frames on PON, combines low cost Ethernet devices with low cost passive optical components, and has attracted much research and deployment attention. IEEE 802.3 Ethernet in the First Mile (EFM) Task Force specified EPON a symmetric upstream/downstream link rate of $1 \mathrm{Gbps}$ and a transmission distance of 10 to $20 \mathrm{~km}$ with 16 ONUs per OLT in the year of 2004. GPON- Gigabit-capable PON, using Generic Framing Procedure to support more efficient packetized traffic transmission, is further developed from APON and BPON by Full Service Access Network (FSAN) consortium, and has increased the transmission rate up to $2.5 \mathrm{Gbps}$ downstream and $1.25 \mathrm{Gbps}$ upstream from hundreds of Mbps downstream and tens of Mbps upstream, respectively.

Time division multiplexing (TDM) technique, which enables multiple users to share a wavelength bandwidth resource in the time domain, is employed in all the above mentioned PONs, i.e., in APON, BPON, EPON and GPON, and they are called TDM PONs. As the number of users increase, and more highbandwidth demanding applications appear, such as $3 \mathrm{D} \mathrm{TV}$, interactive games, tele-medicine, the TDM PONs with a shared transmission rate for each ONU limited to a few tens of Mbps, may not be able to provide the desirable access network services. Therefore, various approaches were proposed in order to increase PONs bandwidth capacity. One of the approaches is to increase the line rate of TDM PONs. E.g., the IEEE802.3av task force is developing 10 Gbps EPON networks, with symmetric $10 \mathrm{Gbps}$ downstream and upstream line rate. This approach requires all EPON nodes be upgraded with higher bit rate transceivers, which will result in a high upgrade cost.

Another approach to increase PON network bandwidth capacity is to employ wavelength division multiplexing (WDM) technique, resulting in WDM PON, which enables multiple wavelengths instead of one wavelength to operate in the PON over the same fiber infrastructure. WDM PON provides one unique wavelength to each ONU, contrast to only one wavelength shared by all ONUs in the TDM PON. But it supports fewer users in a WDM PON, limited by the number of available wavelengths in the PON even with the dense WDM technique, while one user may not consume a whole wavelength's Gbps bit rate with most of present applications. It also incurs high maintenance and inventory cost as each ONU requires a pair of specially tuned transmitter and receiver.

A combined WDM and TDM techniques applied in the PON results in WDM-TDM PON, in which a number of wavelengths are deployed and each wavelength is shared through TDM among several ONUs rather than being dedicated to a single ONU as in the WDM PON case. WDMTDM PON therefore presents an economical upgrade step from TDM PON to WDM PON by offering both high bandwidth and high resource utilization efficiency. In the WDM-TDM PON architecture, if ONUs are pre-configured into a group to share a wavelength, then we call it static WDM-TDM PON; if the ONUs can be dynamically configured into a group to share a wavelength in responding to traffic load changes, then we call it hybrid WDM-TDM PON.

The design and deployment of hybrid WDM-TDM PON face new challenges. Its architecture should allow wavelengths to be assigned to different ONUs dynamically, and transmitters and receivers should be tuned to newly assigned wavelengths in time for packet sending and receiving, and traffic scheduling algorithms should be able to allocate dynamically not only wavelengths but also timeslots to the ONUs based on traffic condition and available resources, i.e., performing dynamic wavelength and bandwidth allocation (DWBA). In this paper, we review the hybrid WDM-TDM PON architectures, transceiver technologies, and especially the DWBA algorithms following a brief introduction of basic EPON and WDM PON 
technologies. We also discuss the physical constraints needed to be considered while developing DWBA algorithms.

\section{EPON AND WDM PON ARCHITECTURES AND ALGORITHMS A. EPON architecture and DBA algorithms}

The general network architecture of the PON network is of a point-to-multipoint nature. It employs a passive fiber tree topology to connect users, i.e., a splitter splits the signal power from a feeder fiber to multiple distribution fibers, and no active power consuming elements are placed in the field. The general TDM PON architecture is shown in Fig. 1.

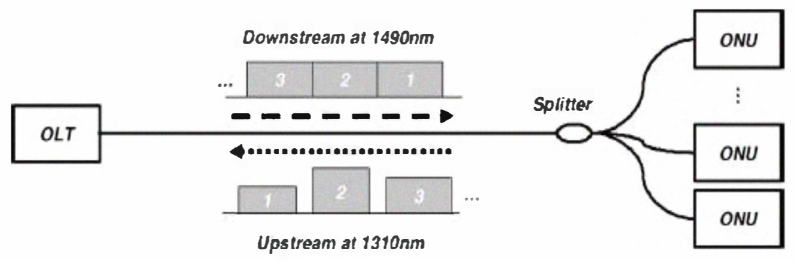

Fig. 1. TDM PON Architecture [1]

The optical line terminal (OLT) and the optical network unit (ONU) are deployed at the two ends of the passive optical distribution network. The passive fiber tree infrastructure and its bandwidth are shared among the users, and TDM technique is required in PON to provide multiple access capability. A central office (CO) (may also refer to OLT) in the PON arbitrates the timeslots distribution among all the users. IEEE 802.3ah task force designed MultiPoint Control Protocol (MPCP) to facilitate the medium access control, and the discovery and registration of ONUs. The MPCP consists of five messages. REGISTER_REQ, REGISTER, and REGISTER ACK are used for the discovery and registration of new ONUs. REPORT and GATE are used for facilitating centralized medium access control. The REPORT message is used to report the instantaneous queue occupancies at an ONU to the OLT, and then OLT makes the decision on the transmission windows for the ONUs based on REPORT messages and certain bandwidth allocation (BA) algorithm, and sends the grants to ONUs through the GATE messages.

The BA algorithms are not specified in MPCP. They are vendor specific in order to meet various users' requirements and desirable network's performance. The algorithms can be classified into static bandwidth allocation (SBA) and dynamic bandwidth allocation (DBA). With SBA, each ONU is allocated a timeslot with a fixed length, which does not require bandwidth negotiation and is thus simple to implement. However, due to the bursty nature of the network traffic, it may result in a situation in which some ONUs with light traffic load have un-occupied timeslots, while other with heavy traffic load have no enough timeslots to handle the traffic in time, resulting in inefficient bandwidth resource utilization. With DBA, the OLT dynamically allocates a variable timeslots to each ONU based on the instantaneous bandwidth demand of the ONU. One of the approaches used for DBA is polling. The OLT polls ONUs and grants timeslots to each ONU in a roundrobin fashion. The timeslots granted to an ONU is determined by the queue status reported from that ONU. With polling, the OLT can dynamically allocate bandwidth for each ONU and flexibly arbitrate the transmissions of multiple ONUs, which can significantly increase bandwidth utilization and improve network performance [2].

More on the PON network architectures and algorithms can be found in survey papers $[1,3,4,5]$.

\section{B. WDM PON architecture}

In a general WDM PON architecture, the OLT has an array of transmitters and receivers, and a separate wavelength channel from the OLT to each ONU, for each of the upstream and downstream directions. This approach creates a point-topoint link between the OLT and each ONU, which differs from the point-to-multipoint topology of the TDM PON. In the WDM PON, each ONU can operate at a rate up to the full bit rate of the wavelength channel and without facing resource competition among the ONUs. Fig.2. shows a typical WDM PON configuration [6].

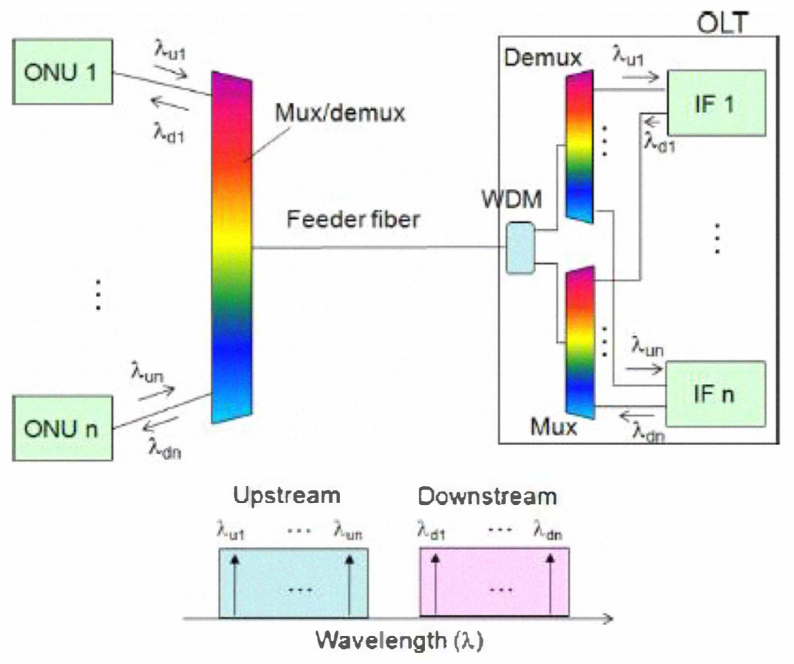

Fig. 2. Typical WDM PON Architecture [6]

In Fig. 2, ONU k ( $k=1$ to $n)$ emits the upstream signal with wavelength $\lambda_{\mathrm{uk}}$ and receives the downstream signal with wavelength $\lambda_{\mathrm{dk}}$. To achieve the wavelength multiplexing of upstream signals from $\lambda_{\mathrm{u} 1}$ to $\lambda_{\mathrm{un}}$ as well as the wavelength demultiplexing of downstream signals from $\lambda_{\mathrm{d} 1}$ to $\lambda_{\mathrm{dn}}$, a wavelength splitter/router is typically used as the optical branching device instead of the power splitter used in TDMbased PON systems. The OLT hosts $n$ interface cards (IF 1 to n) and provides a point-to-point communication between IF $\mathrm{k}$ and ONU k. Papers $[7,8]$ presented survey on WDM PON technologies.

\section{HYBRID WDM-TDM PON ARCHITECTURES}

Hybrid WDM-TDM PON is defined as a PON in which several wavelengths can be used in each direction to establish communication between the OLT and a number of ONUs, each wavelength can be shared by several ONUs, and ONU wavelength assignment can be dynamically changed during operation. In the hybrid WDM-TDM PON, the ability to allow each ONU to access different wavelengths, i.e., to join a logically different TDM PON, facilitates the load balancing 
among the PONs, i.e., when congestion occurs, an ONU is able to join another PON carrying light traffic load. In this section, we review previous WDM-TDM PON work, and discuss hybrid WDM-TDM PON enabling technologies, i.e., wavelength tunable technologies. We also present our work on the WDM-TDM PON network design and experimentation.

\section{A. Previous Work on Architectures}

Authors of paper [9] proposed a hybrid WDM-TDM PON, named SUCCESS-DWA PON, which employs dynamic wavelength allocation to provide bandwidth sharing across multiple physical PONs. Fig.3. shows the network architecture of the SUCCESS-DWA PON. It employs tunable lasers (TLs) and the arrayed waveguide gratings (AWG) at the $\mathrm{CO}$, while WDM filters and a burst-mode receiver are employed within the ONUs. The upstream and downstream traffic is separated by a wideband WDM filter residing between the AWG and the PON. This architecture requires that all wavelengths from the OLT can reach all ONUs across separate physical PONs. TL1 can reach PON1 through AWG channels $\{1,5,9, \ldots\}$; it can also reach PON2 through channels $\{2,6,10 \ldots\}$, and likewise for PON3 and PON4. So, a TL can communicate with any user on a particular PON by determining what wavelength falls in the passband of the user and what wavelength exits the AWG toward that particular PON. The architecture provides scalability by initially deploying one TL and one AWG in the $\mathrm{CO}$, which services multiple subscribers across several PONs. As the demand grows, the architecture can be scaled by adding more TLs to the AWG or by adding another AWG along with more TLs, as shown with the dotted AWG in Fig. 3. The design can provide a flexible dynamic bandwidth allocation capability. However, the architecture is both complicated and expensive. All transmitters in $\mathrm{CO}$ will have to be tunable lasers to achieve the dynamic bandwidth allocation capability.

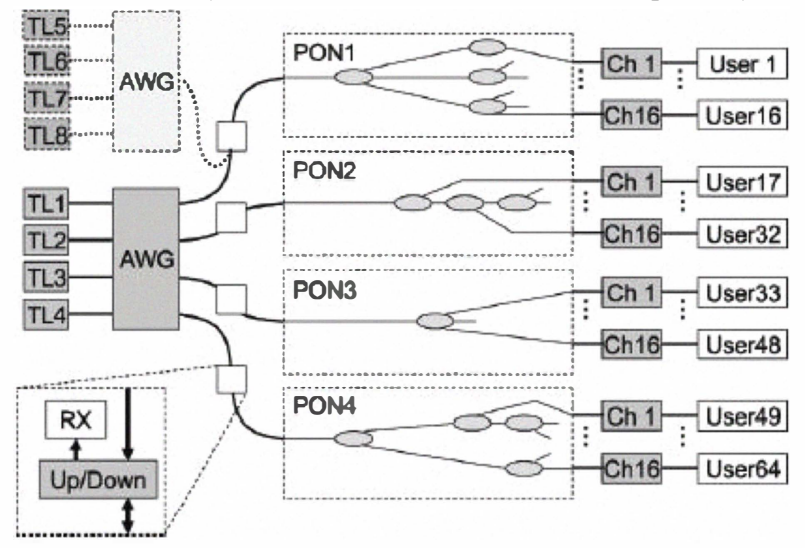

Fig. 3. SUCCESS DWA PON architecture

Other proposed WDM OLT structure in [10] consists of a multicarrier generator and supplies hundreds of optical carriers, thus greatly reducing the number of required laser diodes at the WDM OLT. Each ONU is assigned a separate pair of dedicated upstream and downstream wavelength channels. To decrease costs, ONUs deploy no light source, but simply modulate the optical carriers supplied by the OLT for upstream transmission. This remote modulation scheme realizes wavelength independent ONUs, resulting in reduced costs and simplified operation and maintenance. Note that in the proposed architecture each pair of wavelength channels is dedicated to a different ONU. Thus, upstream wavelength channels are not shared among ONUs and no WDM DBA algorithm is performed. More works on WDM-TDM PON can be found in [11-15].

\section{B. Hybrid WDM-TDM PON Enabling Technologies}

Hybrid WDM-TDM PON requires the transmitters and receivers in the ONUs be able to dynamically and swiftly tune to newly assigned wavelength in order to achieve the wavelength and timeslot resource sharing advantages. There are many works on how to realize the 'colorless' ONU, i.e., a wavelength-independent ONU. Among the colorless schemes, the tunable wavelength transceiver scheme, and injection locking and wavelength seeding schemes are discussed in the following [6,7].

\section{Wavelength Tuning Scheme}

The keys to realizing the dynamic WDM-TDM PON systems are to realize a wavelength-tunable transmitter as well as a wavelength selectable receiver, especially for the low-cost ONUs in access network applications. A fast tuning/selecting speed, e.g., several nanoseconds to several tens of nanoseconds, is quite attractive in terms of flexibly assigning both wavelength and time-slot resources in the same order as set by dynamic bandwidth assignment (DBA) in current TDM based PON systems. Employing a wavelength tunable laser in each ONU is the simplest way to unify the specification of all ONUs, i.e., to realize the colorless ONU. However, the key challenge is to realize a wavelength-tunable ONU that has reasonable cost for access network applications.

\section{Injection-Locking and Wavelength Seeding Schemes}

In the WDM-TDM PON that uses either the injectionlocking or wavelength-seeding scheme, a broadband light source (BLS) or a multi-wavelength light source (MWL) is employed in the OLT as the centralized light source for the upstream (US) signals of all ONUs. The optical spectrum is sliced by mux/demux into multiple lightwaves: each lightwave is continuous wave $(\mathrm{CW})$ having a different central wavelength. In the injection-locking scheme, a Fabry-Perot laser diode (FP-LD) is used as the transmitter of each ONU. The sliced lightwave is injected into the FP-LD, so that the laser wavelength is locked to the wavelength of the injected lightwave. By directly modulating the FP-LD, each ONU can send an upstream signal with appropriate wavelength, which dispenses with the need for ONU-specific LDs. In the wavelength-seeding scheme, a reflective semiconductor optical amplifier (R-SOA) is used as the transmitter of each ONU. The sliced lightwave is fed into the R-SOA, so that the lightwave is amplified and modulated by the upstream signal, and sent back to the mux/demux over the same fiber.

The development and demonstration of colorless ONUs work can be found, e.g., in $[12,16]$ 


\section{Hybrid WDM-TDM PON Using Novel Modulation Techniques}

We proposed a novel hybrid WDM-TDM PON using downlink differential phase shift keying (DPSK) and uplink remodulated on-off keying (OOK) signals based on a shared delay interferometer (DI) and an $N \times N$ cyclic arrayed waveguide gratings (AWG)[17]. Our scheme mitigates the downlink-to-uplink crosstalk, thus improving the performance of uplink remodulated signals. Meanwhile, it replaces the dedicated DIs at each ONU with a shared DI at remote node (RN), thus allowing the ONU receiver to employ just a simple photodetector. Experiments of the 10-Gb/s downlink and 1.25$\mathrm{Gb} / \mathrm{s}$ uplink transmissions verify our proposed scheme.

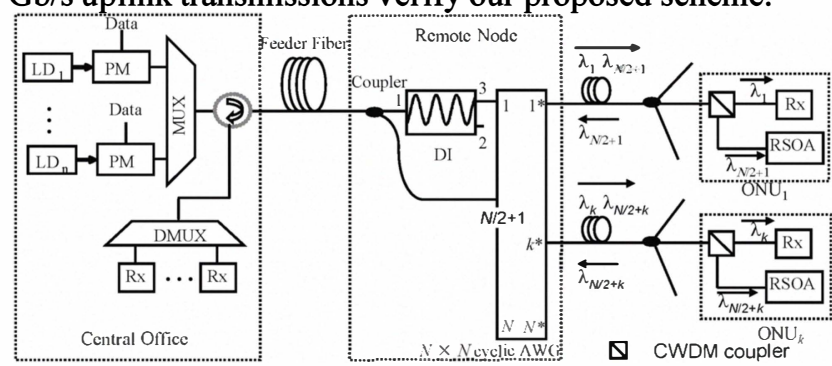

Fig. 4. Novel hybrid WDM-TDM PON architecture

Fig. 4 shows our proposed novel hybrid WDM-TDM PON scheme. In central office (CO), downlink DPSK signal was generated using a phase modulator (PM). The downlink and uplink channels are multiplexed/demultiplexed by a $1 \mathrm{x} N$ multiplexer (MUX) and a demultiplexer (DMUX), respectively. A 3-port optical circulator is used to separate uplink and downlink channels. The multiplexed downlink DPSK signals are sent to remote node (RN) via a feeder fibre. The RN consists of a 1x2 optical coupler, a DI and an $N \times N$ cyclic AWG. The downlink DPSK signals are separated into two parts via the 3-dB optical coupler. One part is sent to the DI for downlink DPSK signals demodulation and the other part is sent to the $N \times N$ cyclic AWG directly for uplink injectionlocking. The demodulated downlink wavelength channel, and $\lambda_{k}$, and the downlink DPSK wavelength channel, and $\lambda_{N / 2+k}$, are then routed to the output port $k^{*}$ of the AWG and distributed to the $\mathrm{ONU}_{k}$ ( $k$ from 1 to $N$ ) via optical splitters and a distribution fibre. At ONU side, a Coarse WDM (CWDM) coupler is used to separate these two wavelength channels. The demodulated downlink DPSK signal is sent to a receiver for downlink data detection. The other part is used as a seeding light to inject into an RSOA for uplink modulation.

\section{HYBRID WDM-TDM PON DWBA ALGORITHMS}

Whereas in TDM single-channel PONs the DBA scheme is focused on scheduling the transmissions on the single wavelength channel, in hybrid WDM-TDM PONs the DBA scheme is expanded to scheduling the transmissions on the different wavelengths supported by the ONUs. In other words, in hybrid WDM-TDM PONs, the algorithms not only schedule the transmission starting time and duration, but also specify on which wavelength channel the transmission should take place, i.e., DWBA, to take the full advantages of the unique features of the network architecture.

Most of the proposed algorithms are on the dynamically scheduling for up-stream traffic from ONUs to OLT as there will be packet collision on the shared uplink channel when multiple ONUs transmit packets simultaneously. While the downlink bandwidth resource allocation schemes are considered relatively simple, since only the OLT in the TDM PON transmits (broadcasts) the packets to all ONUs on the shared downlink.

\section{A. Upstream traffic scheduling}

The upstream traffic scheduling schemes can be classified in two broad paradigms: online and offline scheduling.

Online Scheduling - a given ONU is scheduled for upstream transmission as soon as the OLT receives the REPORT message from the ONU. In other words, the OLT makes scheduling decisions based on individual requests and without global knowledge of the current bandwidth requirements of the other ONUs. A basic online scheduling policy for the WDM-TDM PON is to schedule the upstream transmission for an ONU on the wavelength channel available earliest among the wavelengths supported by the ONU. The amount of the bandwidth (i.e., the length of the granted transmission) allocated to an ONU can be determined according to any of the existing DBA mechanisms for singlechannel TDM PONs.

Offline Scheduling - the ONUs are scheduled for transmission once the OLT has received current MPCP REPORT messages from all ONUs, allowing the OLT to take into consideration in the scheduling the current bandwidth requirements from all ONUs. Since an offline scheduler makes scheduling decisions for all ONUs at once, all of the REPORTs, which are usually appended to the end of the data stream of a gated transmission window, from the previous cycle must be received.

Authors in their papers $[18,19]$ investigated WDM-TDM PONs dynamic wavelength and bandwidth allocation (DWBA) algorithms. It assumes that every ONU is equipped with a fast tunable laser with a tuning speed in the range of microseconds. This architecture enables the ONU to tune its upstream transmission from one wavelength to another at different times depending on the DWBA algorithm deployed at the OLT. Here, the wavelength and bandwidth resources act as a pool, and all ONUs share these resources. There is a granting cycle, in which all ONUs have a chance to transmit data / send REPORTs to the OLT. The authors proposed three variants of a dynamic wavelength and time bandwidth allocation scheme. The first (DWBA-1) schedules ONUs after all REPORT messages have been received for a cycle. Further, DWBA-1 incorporates "fair" distribution of excess bandwidth. The second (DWBA-2) schedules underloaded ONUs upon receipt of their REPORT message and overloaded ONUs after receiving all ONU REPORT messages. When limiting grant sizes and distributing excess from ONUs which do not fully utilize their guaranteed minimums, all REPORTs must be received in a cycle in order to know the excess in that cycle. 
The third (DWBA-3) schedules all ONUs upon receipt of their REPORT messages. Since the OLT needs to grant excess bandwidth, the authors create two GATE messages in this approach. As soon as the ONU REPORT is received, one message grants the guaranteed minimum; and after all REPORTs have been received, another message grants the excess assigned to that $\mathrm{ONU}$.

A scheduling scheme that comes in between the online scheduling and offline scheduling, named online Just-in-time scheduling was proposed in [20]. When a wavelength becomes available, the ONUs that have sent REPORTs are scheduled together according to the selected scheduling policy across all wavelengths. The ONUs that are scheduled would start their transmissions shortly. The OLT transmits GATE messages to these ONUs to inform them of their granted transmission window. The remaining ONUs are in the scheduling pool for the next scheduling round. Alternatively, all ONUs can always be firmly scheduled. The online JIT scheduling framework gives the OLT more opportunity to make better scheduling decisions than standard online scheduling.

The authors in [21], by noting the different network usage behaviors, developed a scheme in which users with complementary behaviors are assigned to the same wavelength to share the network resources so that high channel utilization can be achieved for most of the day, with reduced channels and cost.

\section{B. Downstream traffic scheduling}

Authors of paper [11] discussed downstream traffic scheduling. In their network architecture, each ONU has an extra shared downstream wavelength and has two receivers, one for dedicated wavelength and the other for shared wavelength. The number of ONUs that shares the shared wavelength can be different according to the ONU's bandwidth request. A flow scheduler at the OLT is used for separating and dispatching service flows from the backbone network to dedicated or shared queue, depending on the instantaneous network traffic pattern. The ONU is equipped with a CWDM filter to separate the dedicated and shared wavelengths. The flow scheduler investigates the status of every queue periodically. If the current size of the dedicated queue assigned to a specific ONU exceeds the preset threshold value, and the shared wavelength is available, then some flows to that $\mathrm{ONU}$ are redirected to the shared queue. But these flows will be returned back to the original dedicated queue once the shared queue size becomes less than a certain threshold value.

Multicast traffic scheduling was discussed in [22]. The studied network architecture exploits the WDM dimension by conveying $\mathrm{W}$ wavelengths between the OLT and ONUs. The OLT can transmit/receive data on all wavelengths since it is equipped with dedicated transceivers on each wavelength. It is assumed that each ONU is equipped with a slowly tunable transceiver that enables the ONU to select the most appropriate wavelength to match traffic conditions, and also assume that several end-users are connected to each ONU, so that each ONU in general can request to receive multiple multicast flows. Due to these assumptions, it becomes necessary to duplicate each multicast flow on each WDM channel where an ONU demands it. To avoid as much as possible flow duplication, ONUs that have requested the same multicast flows should tune their receivers to the same wavelength. The authors formalized a Mixed Integer Linear Programming (MILP) problem to find a solution for grouping the ONUs such that the downstream traffic over the available WDM channels minimizes multicast flow duplication.

\section{DISCUSSION ON FUTURE WORK}

The design and deployment of hybrid WDM-TDM PON should consider a smooth upgrading from TDM PON as higher bandwidth demanding applications appear and more bandwidth is needed. The architecture should be flexible that it allows ONUs with higher bandwidth requirements to dynamically select/switch to a wavelength, i.e., to join a TDM PON, where its transmission requirements can be better met. The colorless transceivers, to some extend, determine the cost-effective deployment and performance of the hybrid WDM-TDM PON. The tuning speed and signal transmission quality of the transceivers directly affect the scheduling algorithm operations.

In the hybrid WDM-TDM PON, one ONU may serve a group of users, and each user in the group may have different traffic transmission requirements. One user may upload files, e.g., web server responding to web requests, i.e., mainly contributes to upstream traffic. Another user may download files, e.g., viewing video, IPTV, web browsing, i.e., mainly contributes to downstream traffic. So it needs to consider the traffic transfer for this group of users (connected to one ONU) in both directions at the same time, i.e., both upstream traffic and downstream traffic are scheduled simultaneously.

Physical constraints also need to be considered in developing the algorithms. If the transmitter in the ONU is wavelength tunable one, then whichever wavelength to use to transfer the traffic will not affect the upstream or downstream algorithms performance. As the transmitters can tune to any available wavelengths, the selection of upstream wavelength will have nothing to do with the selection of downstream wavelength. But the reality is that even a tunable transmitter is used (at a high cost today), the selection of upstream/downstream wavelength still has its constraints, e.g., there is a limitation of transmitter's tuning range. If the injection-locking or wavelength seeding techniques are used, and furthermore, wavelength-reuse is requested, which uses a single wavelength light source in OLT for cost reduction and implementation simplicity, then the same wavelength is assigned to one ONU for both upstream and downstream transmission, i.e., the assignment of upstream wavelength and downstream wavelength can not be independent. The existing hybrid WDM-TDM PON DWBA algorithms were developed without considering the interconnection/dependence between the upstream wavelength and downstream wavelength, and the upstream and traffic downstream traffic were scheduled separately . In practice, there is a need for algorithms that schedule both the upstream and downstream traffic at the same time under the physical constraints. 


\section{CONCLUSION}

The paper gave a brief introduction of PON technologies, and reviewed the current works on hybrid WDM-TDM PON architecture design and DWBA algorithms development. Hybrid WDM-TDM PON presents a flexible and cost-effective optical access network solution, but for practical deployment there are still many technique challenges faced. One of these key challenges is about the wavelength tuning technique. The wavelength tuning speed and transmission quality of optical transmitters directly affect the network performance. While in the development of DWBA algorithms, the physical constraints should also be taken into consideration.

\section{REFERENCES}

[1] L.G. Kazovsky, W. Shaw, D. Gutierrez, N. Cheng, S. Wong, "Nextgeneration optical access networks", IEEE J. Lightwave Tech., vol.25, no.11, Nov 2007, pp3428-3442

[2] G. Kramer, B. Mukherjee, and G. Pesavento, "IPACT: A dynamic protocol for an Ethernet PON (EPON)," IEEE Commun. Mag., vol. 40, no. 2, pp. 74-80, Feb. 2002.

[3] F. Effenberger, T. El-Bawab, "Passive optical networks(PONs): past, present and future", Journal of Optical Switching and Networking, no.6, 2009, pp143-50

[4] M. P. McGarry, M. Reisslein, M. Maier, "Ethernet passive optical network architectures and dynamic bandwidth allocation algorithms", IEEE Communications Surveys, V10, N3, 2008, pp46-60

[5] J. Zheng, H.T. Houftah, "A survey of dynamic bandwidth allocation algorithms for Ethernet passive optical networks", Journal of Optical Switching and Networking, N6, 2009, pp151-162

[6] J. Kani, "Enabling technologies for future scalable and flexible WDMPON and WDM/TDM-PON systems", to appear in IEEE Journal of Selected Topics in Quantum Electronics", 2010

[7] A. Banerjee, Y. Park, F. Clarke, H. Song, S. Yang, G. Kramer, K. Kim, and B. Mukherjee, "Wavelength-division-multiplexed passive optical network (WDM-PON) technologies for broadband access: a review," J. Opt. Netw., vol. 4, no. 11, pp. 737- 758, Nov. 2005.

[8] M. P. McGarry, M. Reisslein, M. Maier, "WDM Ethernet passive optical networks", IEEE Communications Magazine, V44, N2, Feb 2006, pps18s25

[9] Y.-L. Hsueh, M. S. Rogge, S. Yamamoto, L. G. Kazovsky, “A Highly Flexible and Efficient Passive Optical Network Employing Dynamic Wavelength Allocation", IEEE J. Lightwave Tech., vol. 23, no. 1, Jan 2005, pp.277-286.

[10] D. J. Shin et al., "Hybrid WDM/TDM-PON with Wavelength-SelectionFree Transmitters," IEEE J. Lightwave Tech., vol. 23, no. 1, Jan. 2005, pp. 187-95.

[11] H. Song and B. Mukherjee, Y. Park, S. Yang, "Shared-Wavelength WDM-PON Access Network for Supporting Downstream Traffic with QoS", OFC 2006, OThK2.

[12] Han-Hyub Lee, et al, "First Commercial Service of a Colorless Gigabit WDM/TDM Hybrid PON System", OFC09

[13] G-K. Chang, et al, "Key technologies of WDM-PON for future converged optical broadband access networks", J. OPT. COMMUN. NETW, vol. 1. no.4, 2009

[14] M. Maier, M. Herzog, and M. Reisslein, "STARGATE: the next evolutionary step toward unleashing the potential of WDM EPONs," IEEE Commun. Mag., vol. 45, no. 5, pp. 50-56, May 2007.

[15] M. Maier, "WDM passive optical networks and beyond: the road ahead", J. OPT. COMMUN. NETW, V1. N4, 2009

[16] Z. Belfqih, P. Chanclou, F.Saliou, "Hybrid WDM-TDM Passive Optical Network in burst mode configuration with RSOA", OFC08

[17] X. Cheng, Y.K. Yeo, Z. Xu, Y. Wang, "A Novel Hybrid WDM/TDMPON using Downlink DPSK and Uplink Remodulated OOK Signals Based on a Shared DI", ECOC, 2009

[18] A.R. Dhaini, C.M. Assi, M.Maier, A. Shami, "Dynamic wavelength and bandwidth allocation in hybrid TDM/WDM EPON network", IEEE J. Lightwave Tech., vo.125, no.1, Jan 2007, pp277-286
[19] A.R. Dhaini, C.M. Assi, A. Shami, "Quality of service in TDM/WDM Ethernet passive optical networks(EPONs)", Proceedings of the $11^{\text {th }}$ IEEE Symposium on Computers and Communications (ISCC), 2006

[20] M.P.McGarry, et al, "Just-in-time scheduling for multichannel EPONs", IEEE J. Lightwave Tech., vol.26, no.10, May 2008, pp1204-1216

[21] L. Shi, H. Song, "Behavior-aware user-assignment in hybrid PON planning", OFC2009

[22] M. Alfani, J. M. Finochietto, F. Neri, "Efficient Multicast Bandwidth Allocation in TDM-WDM PONs", OFC 2009, JYhA82 